\title{
Controlled green synthesis of hybrid organo-inorganic nanomaterials based on poly(ethylene terephthalate) and silver nanoparticles by $\mathrm{X}$-ray radiolysis
}

\author{
O. V. Arzhakova ${ }^{1}$, A. A. Dolgova ${ }^{1}$, A. Yu. Yarysheva ${ }^{1}$, A. A. Zezin ${ }^{2}$ \\ ${ }^{1}$ Faculty of Chemistry, Lomonosov Moscow State University, 1/3 Leninskie Gory, 119991 Moscow, Russia \\ ${ }^{2}$ Enikolopov Institute of Synthetic Polymeric Materials Russian Academy of Sciences (ISPM RAS), 70 ul. Profsoyuznaya, \\ 117393 Moscow, Russia
}

Received 27 August 2020; accepted in revised form 28 November 2020

\begin{abstract}
Self-confined synthesis of silver nanoparticles (AgNPs) within mesoporous host matrixes based on poly(ethylene terephthalate) (PET) upon X-ray radiolysis is studied. Mesostructured PET matrixes with a porosity of 35 vol\% and pore dimensions below $10 \mathrm{~nm}$ were prepared and loaded with $\mathrm{Ag}^{+}$ions via the mechanism of environmental crazing. Upon subsequent X-ray irradiation of silver-loaded samples, $\mathrm{Ag}^{+}$ions experience reduction into $\mathrm{Ag}^{0}$ within mesopores. In this case, solvated electrons, alcohol radicals, and acetaldehyde act as effective reducing agents. The calculations show that the X-ray absorption dose rate for the solutions of silver nitrate $(33.6 \mathrm{~Gy} / \mathrm{s})$ is nearly three times higher than that of pure PET $(10.1 \mathrm{~Gy} / \mathrm{s})$. This contrast allows a selective synthesis of AgNPs within mesopores whereas the dose rate for the PET matrix lies within the level of sterilization. Mesoporous matrixes provide confined conditions for the synthesis of AgNPs with mean dimensions of $\sim 2-3 \mathrm{~nm}$ and also serve as a stabilizing medium that prevents their aggregation and spares the use of any capping agents. This reagent-free approach offers a new route for preparing diverse hybrid organo-inorganic nanomaterials with desired functional properties.
\end{abstract}

Keywords: nanocomposites, hybrid organo-inorganic nanomaterials, X-ray radiolysis, silver nanoparticles, mesoporous polymers

\section{Introduction}

Preparation of novel hybrid organo-inorganic nanomaterials (HOIN) with controlled morphology and desired functional properties, including antibacterial and plasmonic properties, conductivity, catalytic activity, etc. $[1,2]$ presents the challenging direction of materials science. The demands for innovative HOINs are dictated by the needs of agriculture, medicine, renewable energy, textile industry, health care, waste-water treatment, nano-barcode technology, food sector, etc. [3-6]. Scientific studies in this direction are focused on the design of advanced nanomaterials with improved functional and mechanical properties, and the search for alternative cost-effective and sustainable methods for their preparation with the account for environmental concerns is spurred [7]. According to general knowledge, typical HOINs are composed of an organic matrix loaded with an incorporated inorganic component. Basic requirements for modern HOINs are the following: high performance, controlled distribution of inorganic nanoparticles (NPs) without their aggregation, high (surface area)-to-volume ratio, high content of active surface atoms, high level of dispersion of both phases, stability, and fair mechanical properties. Different strategies have been used for the synthesis of

\footnotetext{
${ }^{*}$ Corresponding author, e-mail: arzhakova8888@gmail.com (C) BME-PT
} 
inorganic NPs and the preparation of HOINs [8]. This work highlights the advantages of X-ray irradiation as an ecologically safe and reagent-free strategy for the development of metal nanoparticles within mesoporous organic matrixes [9-15]. For the first time, this approach has been used for the surface treatment of a Langmuir monolayer leading to the formation of nanoparticles in bulk aqueous solutions $[16,17]$. Further development of this approach involves the synthesis of functional structures and metallic/bimetallic nanoparticles [12, 15, 16, 18-23], nanocomposites containing nanoparticles of transition and noble metals within inter-polyelectrolyte complexes, and reduction of metal ions within polyacrylic acid (PAA)-polyethyleneimine systems [15]. However, in all cases, undesirable aggregation of nanoparticles presents the main challenge, and this problem can be solved by the use of diverse capping agents $[11,24,25]$.

To the best of our knowledge, information on the preparation of sustainable HOINs via controlled Xray-induced reduction of metal ions within mesoporous nanostructured matrixes based on commercial polymers is missing. When the reduction of metal ions is performed under confined conditions, one can expect that the mesoporous polymer matrix by itself can serve as an 'efficient' capping agent for NPs that prevents their aggregation. Moreover, in this case, the polymer matrix can also serve as a solid framework, thus providing fair mechanical properties of the resultant HOINs.

Environmental crazing (EC) offers an efficient strategy for the preparation of sustainable mesoporous polymeric materials with pore dimensions below $10 \mathrm{~nm}$. Environmental crazing presents a specific mode of plastic deformation of solid polymers in the presence of physically active liquid environments (PALE), which is accompanied by the development of porosity as crazes with their unique fibrillarporous structure [26-31]. The resultant mesostructured materials can be used as efficient host matrixes for the preparation of HOINs via bottom-up development of NPs through in situ reduction of metal ions within nanoscale pores. Of special importance is the fact that this approach allows the use of lowcost commercial polymers such as polyethylene, polypropylene, poly(ethylene terephthalate) (PET), etc. In this study, the choice of PET was dictated by the fact that this polymer is widely used in industry and daily life: production of bottles for soft drinks
(30\%) [32] and household chemicals, textiles and yarns, packaging trays, and blister packs, safe packaging, including clamshells, cleaning cloths, waterproofing barrier in undersea cables, etc., thus creating serious ecological challenges. Hence, search for new safe routes allowing the preparation of innovative PET-based materials with valuable functional properties is highly motivated.

This work addresses the preparation of hybrid PETbased nanomaterials containing silver nanoparticles, which presents an evident interest from the viewpoint of nanotechnology and materials science as silver nanoparticles show remarkable size and shapedependent optical properties as well as excellent antibacterial properties due to the therapeutic action of silver ions even on antibiotic-resistant bacteria [33]. These PET/Ag materials can be used in various applications, including bandages and cloths, face masks for protection against bacteria and viruses, materials for health care, catalysis, photonics, cosmetics, etc. [34].

This work advances a new ecologically safe strategy for the preparation of HOINs based on PET and silver nanoparticles via X-ray radiolysis: which involves EC for the preparation of mesoporous matrixes and their loading with silver salts and their subsequent reduction to silver NPs within mesopores under confined conditions.

\section{Experimental}

In this study, the films of amorphous glassy PET with a thickness of $100 \mu \mathrm{m}$ (PLASTPOLIMER, Russia) were used. As a PALE providing environmental crazing of PET, isopropanol (IPA) (Aldrich, USA) was used. Mesoporous polymer matrixes were prepared and loaded with silver ions via environmental crazing: the samples with a gage size of $50 \times 30 \mathrm{~mm}$ were stretched at room temperature to a fixed tensile strain $(125 \%)$ in the presence of water-IPA solutions (3:1) containing $5 \mathrm{wt} \%$ of dissolved silver nitrate (Aldrich, USA) via EC. At this tensile strain (125\%), the porosity of EC PET samples is maximum $(\sim 35 \%)$. Porosity $W$ of the PET films was estimated from changes in geometric dimensions of the samples upon tensile drawing as $W=\Delta V /\left(V_{0}+\Delta V\right)$, where $V_{0}$ is the initial volume of the sample, $\Delta V$ is the difference between the volume of the initial sample and the EC sample. The experimental error was $\sim 3 \%$. The content of silver nitrate in the PET films was estimated according to TGA measurements (TA4000 
Mettler thermal analyzer, TG50 unit, USA) and gravimetrically (HR-250AZG AND analytical balance, Japan). Gravimetric and TGA tests were performed for not less than 3-5 samples. The temperature interval (TGA tests) was varied from room temperature to $450{ }^{\circ} \mathrm{C}$. The heating rate was $10 \mathrm{~K} / \mathrm{min}$ (nitrogen atmosphere).

Average pore diameter $D_{\mathrm{p}}$ and fibril diameter $D_{\mathrm{f}}$ of mesoporous PET films were estimated using the pressure-driven liquid permeability (PDLP) method using custom-made equipment and hydrodynamic Hagen-Poiseuille (pore diameter) and D'Arcy-Happel (fibril diameter) hydrodynamic models [31] as well as using the method of low-temperature nitrogen adsorption (LTNA) and permporometry, the basic principle of which is based on capillary condensation of vapor and the blocking effect of permeation of noncondensable gas. PDLP tests were performed for not less than 3-5 samples. The experimental error was $5 \%$. For the estimation of the parameters of the porous structure of the mesoporous PET films, adsorption measurements were performed with an ASAP 2020 automatic analyzer (MicroMeritics, Canada). Before the measurements, all samples were ground and evacuated for 4-5-h. Pore size and volume were estimated according to the $\mathrm{BJH}$ method using the Micromeritics software. BJH method (Barrett, Joyner, and Halenda) is a procedure for calculating pore size distributions from experimental isotherms using the Kelvin model of pore filling [35]. The structure of the PET samples was characterized using an EVO40-XPV scanning electron microscope (Carl Zeiss, Jena, Germany). Prior to SEM observations, the fractured samples were coated with a conducting gold layer with a thickness of 50-70 nm using a Giko IB-3 setup.

To prepare HOINs, the PET films with incorporated $\mathrm{Ag}^{+}$were irradiated in a water-alcohol medium (water-ethanol; 10 vol\% of ethanol) using an X-ray device equipped with a 5-BKnV-6W tube (applied voltage $33 \mathrm{keV}$, the anode current $80 \mathrm{~mA}$ ). The samples were irradiated in plastic cells with a thickness of $5 \mathrm{~mm}$. Effective X-ray energy was estimated to be $\sim 21 \mathrm{keV}$. The above conditions provide a uniform generation of radiolysis products. To avoid the oxidation of silver nanoparticles by air oxygen dissolved in the water-ethanol solution, the solutions were bubbled with high-purity argon. Irradiation time was varied from 10 to $20 \mathrm{~min}$. The absorbed dose rate was estimated using ferrous sulfate dosimeter $(17 \mathrm{~Gy} / \mathrm{s})$. The actual dose rate for each component (water-alcohol medium and polymer film) was calculated with the account for mass absorption coefficients and effective energy of X-rays [36].

The structure of the PET-based HOINs with AgNPs was studied by TEM observations on a Leo-912 AB OMEGA microscope at an accelerating voltage of 60$120 \mathrm{kV}$. Ultrathin sections were cut with a diamond knife (ultramicrotome, Reichert Jung). TEM images were analyzed using a FemtoScan software [31].

\section{Results and discussion}

Traditional protocols for the preparation of HOINs containing metallic nanoparticles either involve the 'top-down' approach when metal is dispersed into small-sized NPs by grinding and then blended with polymer (in a melt or a solution) or the 'bottom-up' approach when NPs are produced by chemical reduction using diverse chemical reagents $[10,14,24,37$, 38]. This work offers a new facile and reagent-free 'bottom-up' approach for the preparation of HOINs via $\mathrm{EC}$, which allows fabrication of unique mesoporous polymer matrixes and simultaneous impregnation of metal ions (stage I) as precursors for their further reduction and X-ray radiolysis of the films loaded with silver ions (stage II).

To gain a deeper insight into the structure of the HOINs, the structure of host mesoporous PET matrixes was studied. Upon EC, the porosity of the PET films increases with increasing the tensile strain. The porosity-(tensile strain) curve passes maximum at a tensile strain of $125 \%$. Figure 1 shows the SEM images of the PET samples with a tensile strain of $125 \%$. Environmental classical crazing involves stress-induced initiation of fibrillar-porous structure within crazes. The inner structure of crazes can be described as an array of nanoscale fibrils spaced by mesopores $[26,31,39]$. The porous structure of the EC PET (pore diameter $D_{\mathrm{p}}$ and fibril diameter $D_{\mathrm{f}}$ ) was studied by the PDLP and LTNA methods and related calculations: $D_{\mathrm{p}}=4.5 \mathrm{~nm}$ and $D_{\mathrm{f}}=3.6 \mathrm{~nm}$. Content of incorporated silver nitrate within the fibrillar-porous crazes was estimated gravimetrically as $2.5 \mathrm{wt} \%$.

After X-ray irradiation for $10 \mathrm{~min}$, colorless PET films become brown, thus suggesting the formation of $\mathrm{Ag}^{0}$. To prove this assumption, the PET/Ag samples after X-ray irradiation were studied by the TEM observations (Figure 2).

As follows from the TEM images (Figure 2), the PET samples are loaded with spherical silver nanoparticles, 


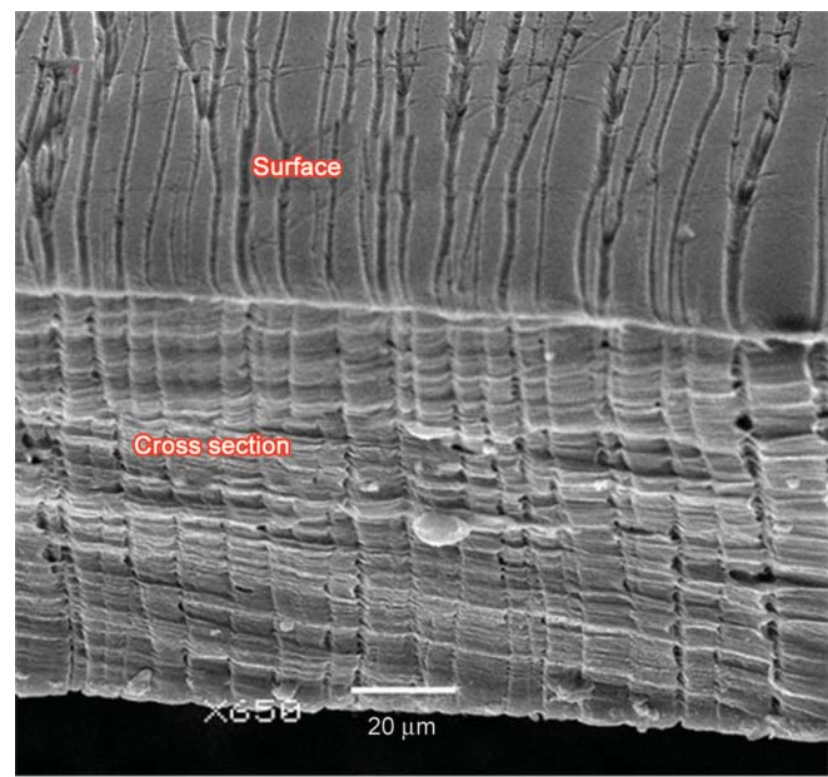

a)

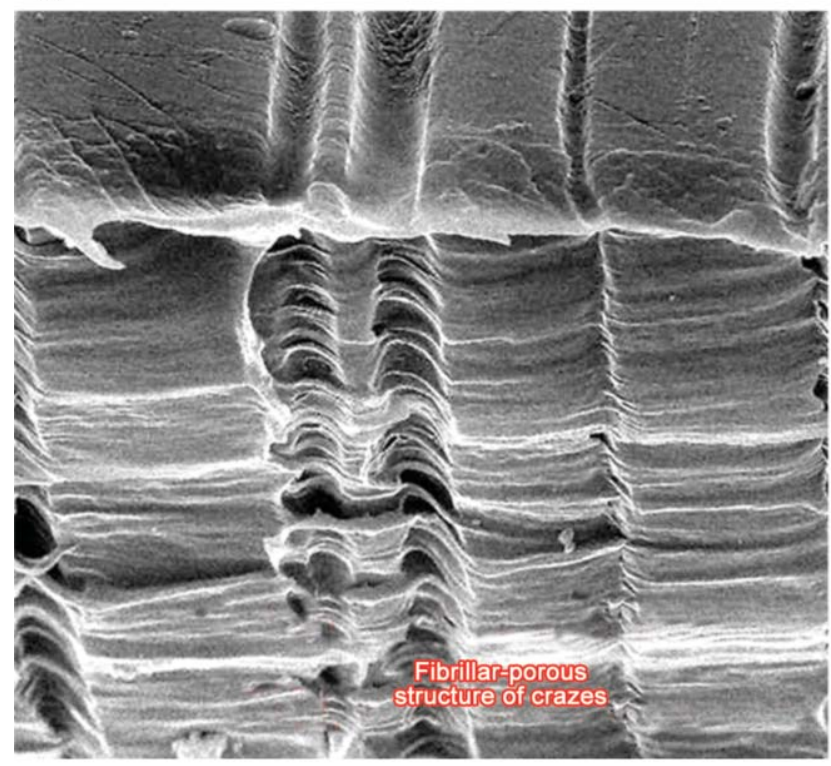

c)

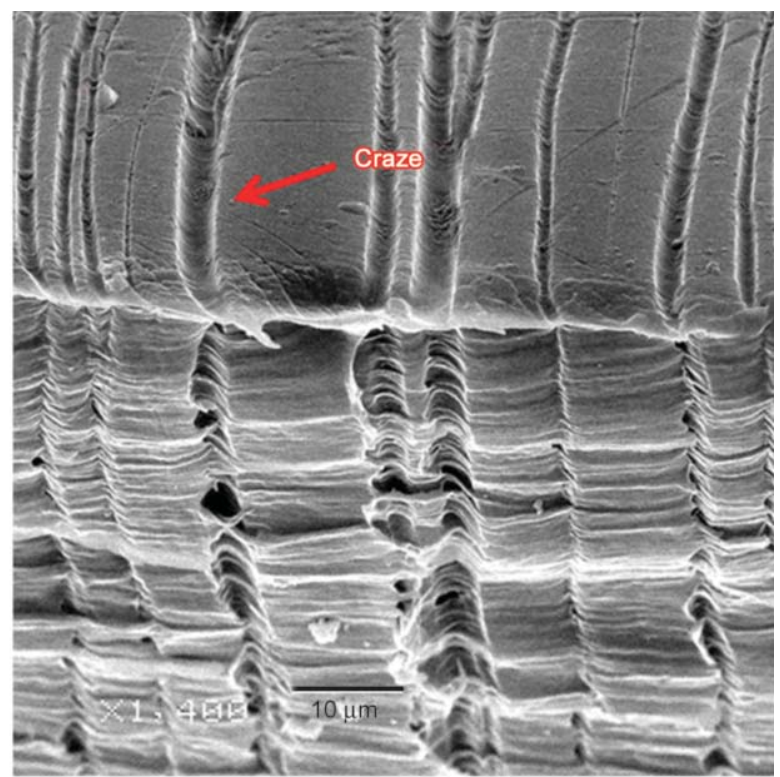

b)

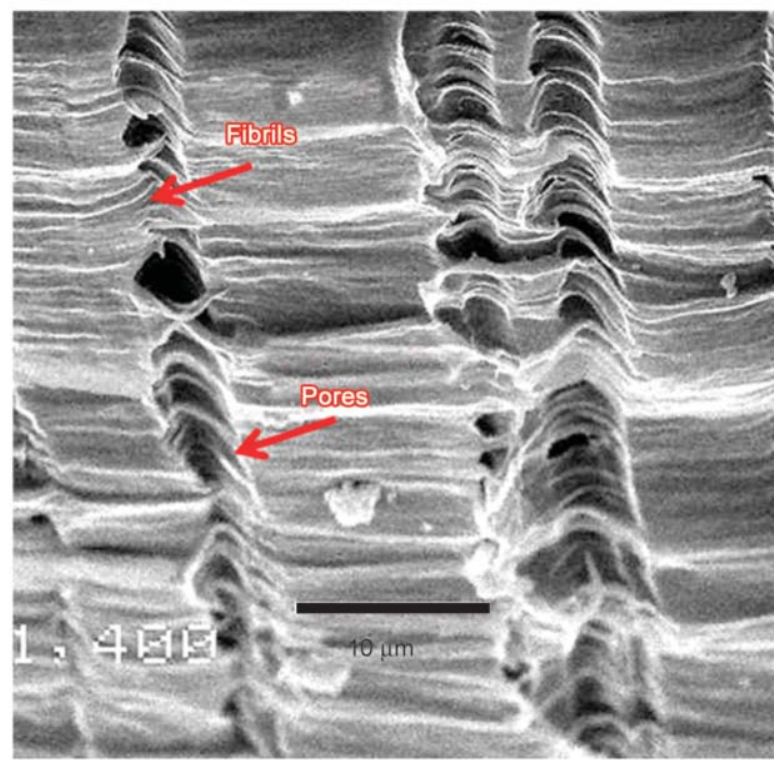

d)

Figure 1. SEM images of the PET films after EC (water-IPA solution) by a tensile strain of 125\%: (a) surface with multiple crazes, (b, c) fractured surface, and (d) internal fibrillar-porous structure of crazes.

which are accommodated within the fibrillar-porous structure of crazes. This observation agrees with the SEM images in Figure 1 when crazes loaded with AgNPs ( 100-200 nm wide) alternate with the regions of bulk polymer ( $\sim 200-350$ nmwide). Figure 3 presents the TEM images and electron diffractogram of the Ag-loaded PET samples as well as the histogram illustrating the size distribution of silver nanoparticles.

Electron diffractogram of the test samples (Figure 3c) unequivocally proves that $\mathrm{X}$-ray radiolysis of the $\mathrm{Ag}^{+}$-loaded PET films in the presence of aqueous alcohol solutions provides an efficient chemical reduction of silver ions to $\mathrm{Ag}^{0}$. The corresponding XRD pattern reveals the formation of the face-centered cubic crystalline phase of silver NPs. The diffraction reflections refer to the (111), (200), (220), and (311) reflection planes, respectively. Interplanar distances of the crystalline lattices are 2.36, 2.04, 1.45, 1.23, and $1.17 \AA$. This evidence fits the standard data reference for silver (ICCD card number: 00-003-0921). Silver nanoparticles with dimensions below $10 \mathrm{~nm}$ are distributed within mesopores of fibrillar-porous crazes without agglomeration (Figures $3 a, 3 b$ ). The size distribution of AgNPs in the resultant HOIN is seen to be narrow and unimodal, with a well-pronounced maximum at $\sim 2 \mathrm{~nm}$ (Figure $3 \mathrm{~d}$ ). Noteworthy is that the average size of silver NPs corresponds 


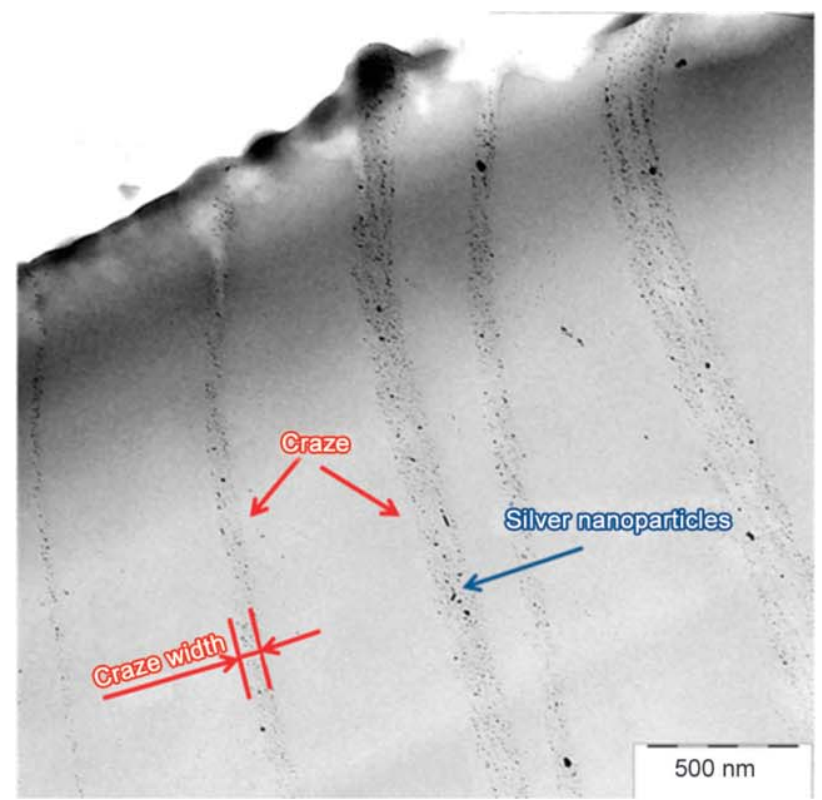

a)

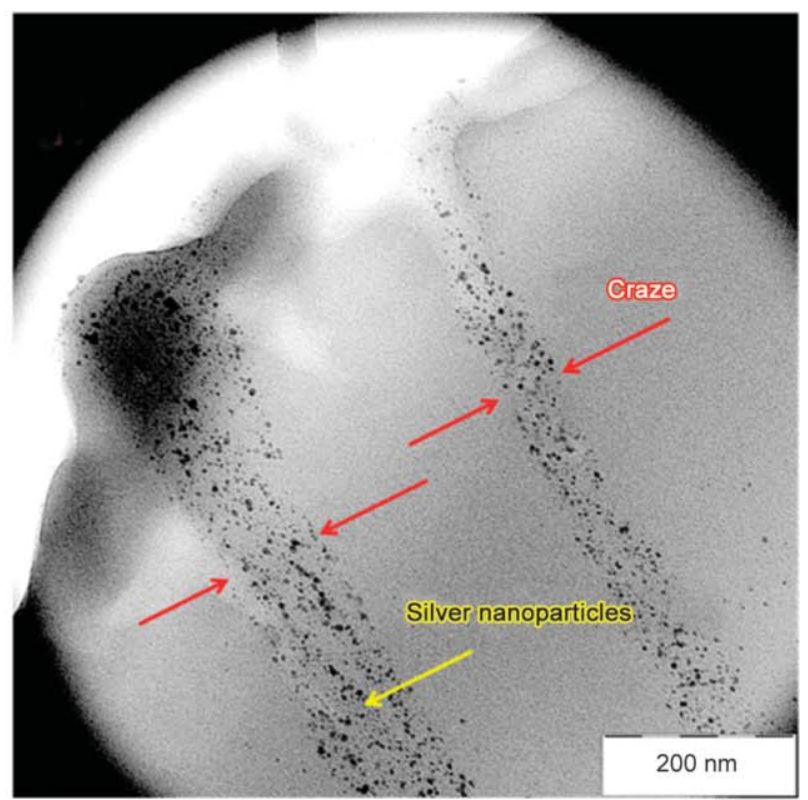

b)

Figure 2. TEM images of thin cross-sectioned samples of the silver-loaded PET films after X-ray irradiation for 10 min (tensile strain $\varepsilon=125 \%$ ). Scale bar: $500 \mathrm{~nm}$ (a) and $200 \mathrm{~nm}(\mathrm{~b})$.

to the pore dimensions of the EC PET samples, and this fact can be explained as follows: reduction of $\mathrm{Ag}^{+}$ions to $\mathrm{Ag}^{0}$ and clustering of $\mathrm{Ag}^{0}$ atoms into AgNPs proceed within the mesopores under confined conditions. Of special importance is the fact that the samples are stable, and the dimensions of silver NPs do not change with time. In this case, the formation of stable silver nanoparticles does not require the use of any capping agents as mesoporous PET matrix effectively prevents aggregation and stabilize the system in whole. Therefore, in situ X-radiolysis of the $\mathrm{AgNO}_{3}$-containing PET films allows reduction of silver ions into $\mathrm{Ag}^{0}$ and preparation of the HOINs containing AgNPs with uniform dimensions.

The effect of irradiation duration on the structure of Ag-containing PET films was studied: the exposure time increases from 10 (10/Ag-PET) to $15 \mathrm{~min}$ (15/Ag-PET) (Figure 4).

As follows from Figures 3 and 4, as the irradiation time increases from 10 to $15 \mathrm{~min}$, the number of AgNPs within fibrillar-porous crazes increases from $20 \cdot 10^{4}$ to $30 \cdot 10^{4} / \mu^{2}$. The corresponding XRD patterns of the 15/Ag-PET samples are similar to those of the 10/Ag-PET samples, but all lines become sharper and better pronounced and bright spots revealing the presence of silver single crystals are observed (Figure 4c). As follows from the histogram (Figure 4d), the full length at half-width of the size distribution sample becomes broader, and its peak is shifted towards higher dimensions (from 2 to $3 \mathrm{~nm}$ ). Hence, as the irradiation time increases, some higher-order nanostructures containing NP clusters are developed. Minor enlargement of AgNPs (by $1 \mathrm{~nm}$ ) suggests that, in whole, reduction of incorporated silver ions to $\mathrm{Ag} 0$ is nearly completed. Noteworthy is that the left-hand tail of the histogram (Figure 4d) becomes longer as new small-sized particles become visible in TEM observations and the existing AgNPs become bigger due to clustering. When the irradiation time increases to $20 \mathrm{~min}$, all patterns are identical to those of 15/Ag-PET samples. This evidence proves that the formation of AgNPs is nearly completed within $15 \mathrm{~min}$.

Let us discuss the mechanism of X-ray radiolysis of $\mathrm{Ag}^{+}$within mesopores of the nanostructured mesoporous PET matrixes with the account for the literature data [40-42]. The products providing the redox process are primarily produced via water radiolysis. Reduction of $\mathrm{Ag}^{+}$upon X-ray radiolysis in the aqueous alcohol solutions proceeds as shown by Equation (1):

$\mathrm{H}_{2} \mathrm{O} \rightarrow e_{\mathrm{aq}}^{-}, \mathrm{OH} \cdot \mathrm{H}_{3} \mathrm{O}^{+}, \mathrm{H}_{2}, \mathrm{H}^{\cdot}$

The principal products of X-ray radiolysis are hydrated electrons and $\mathrm{OH}$ radicals. Hydrated electrons are characterized by high reduction potential $(-2.9 \mathrm{~V})$ [43], while the $\mathrm{OH}$ radicals serve as oxidizing agents. Additives (ethanol or IPA) as $\mathrm{OH}$ radical scavengers 


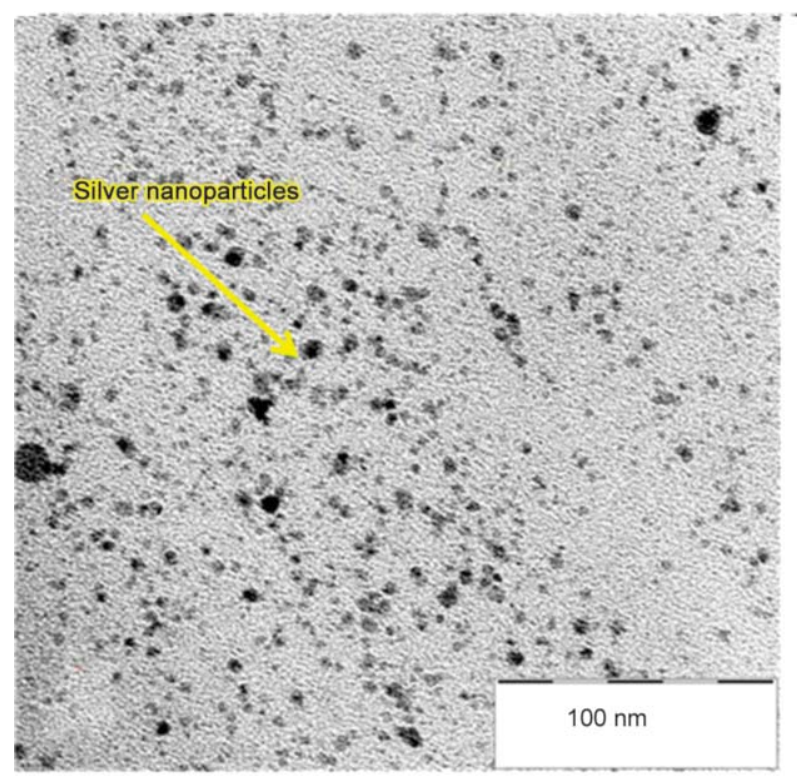

a)

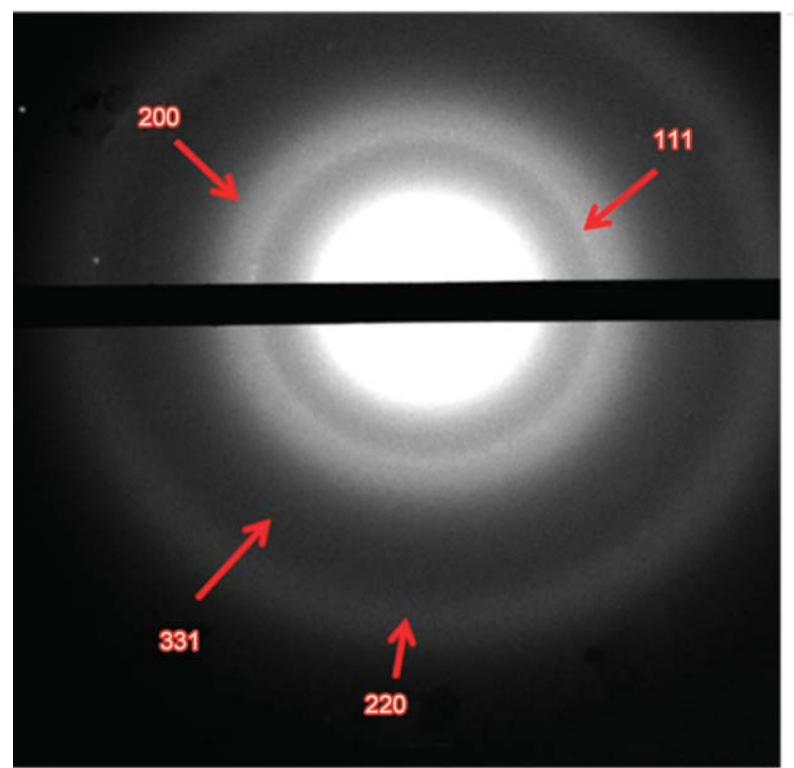

c)

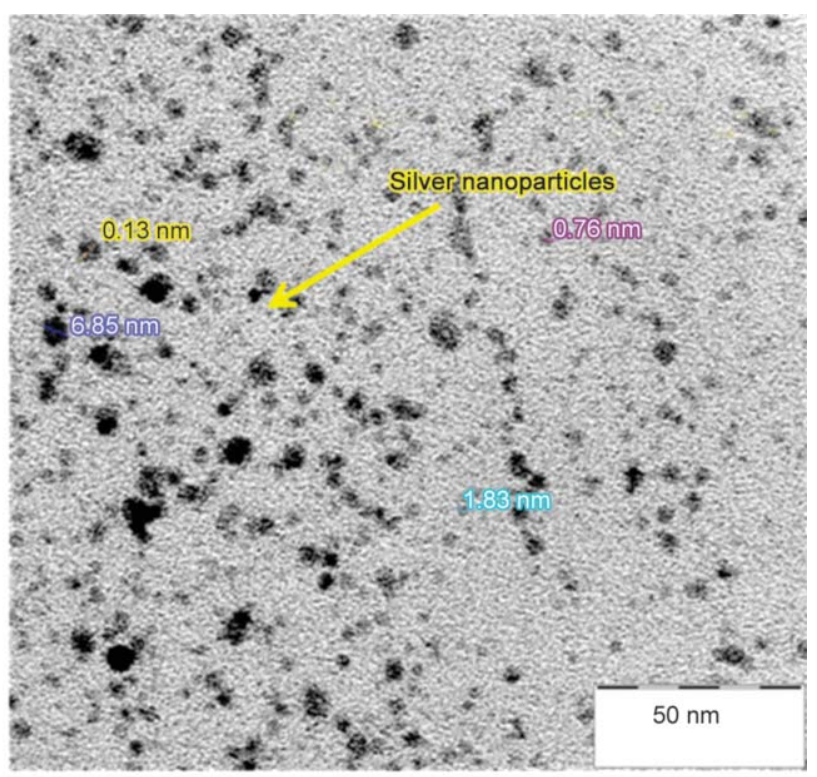

b)

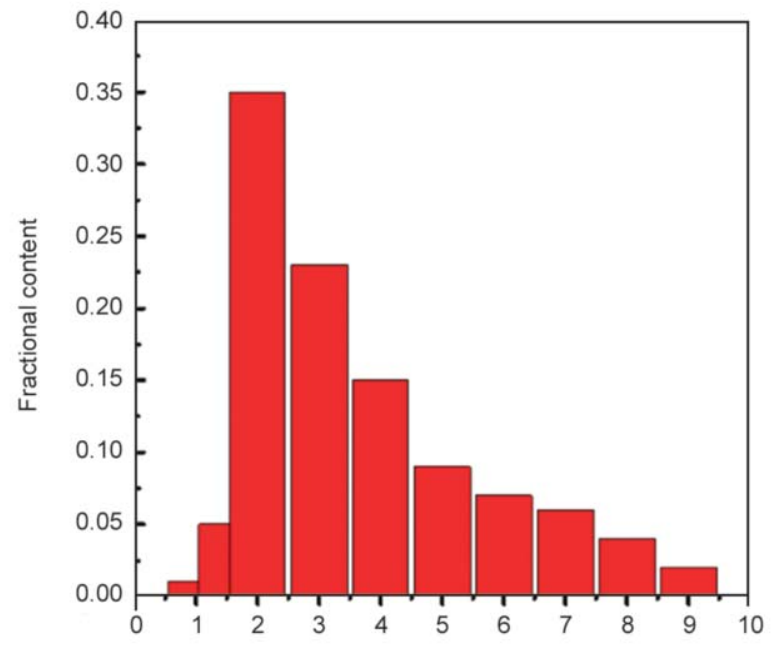

d)

Size $[\mathrm{nm}]$

Figure 3. ( $a, b)$ TEM images of thin sections of the PET films containing AgNPs after X-ray radiolysis (irradiation time is $10 \mathrm{~min}$ ), (c) electron diffractogram of the PET films containing AgNPs; (d) histogram illustrating size distribution of AgNPs.

ensure the generation of reduction agents [11]; see Equation (2):

$$
\mathrm{CH}_{3} \mathrm{CH}_{2} \mathrm{OH}+\mathrm{OH} \cdot \rightarrow \mathrm{CH}_{3} \cdot \mathrm{CHOH}+\mathrm{H}_{2} \mathrm{O}
$$

Alcohol radicals $\mathrm{Me} \cdot \mathrm{CH} \mathrm{OH}\left(\mathrm{E}_{0 \mathrm{Me} \cdot \mathrm{CHOH}}=\sim 1.4 \mathrm{~V}\right)$ are also characterized by strong reduction properties. The first stage involves the reduction of $\mathrm{Ag}^{+}$to isolated silver atoms and the formation of clusters composed of Ag+ and atoms; see Equations (3) and (4):

$$
(\mathrm{Ag})^{+}+e_{\mathrm{aq}}^{-} \rightarrow(\mathrm{Ag})^{0}
$$

$$
(\mathrm{Ag})^{+}+(\mathrm{Ag})^{0} \rightarrow\left(\mathrm{Ag}_{2}\right)^{+} \rightarrow \ldots \rightarrow\left(\mathrm{Ag}_{\mathrm{m}}\right)^{\mathrm{n}+}
$$

Clustering proceeds via the reduction of $\mathrm{Ag}^{+}$on the surface of the primary NPs (Equation (5)) and coalescence (Equation (6)):

$$
\begin{aligned}
& \left(\mathrm{Ag}_{\mathrm{m}}\right)^{\mathrm{n}+}+(\mathrm{Ag})^{+} \rightarrow\left(\mathrm{Ag}_{\mathrm{m}+1}\right)^{(\mathrm{n}+1)} \rightarrow\left(\mathrm{Ag}_{\mathrm{m}+1}\right)^{\mathrm{n}} \\
& \left(\mathrm{Ag}_{\mathrm{m}}\right)^{\mathrm{n}+}+\left(\mathrm{Ag}_{\mathrm{k}}\right)^{\mathrm{g}+} \rightarrow\left(\mathrm{Ag}_{\mathrm{m}+\mathrm{k}}\right)^{(\mathrm{n}+\mathrm{g})^{+}}
\end{aligned}
$$

The critical stage behind the development of AgNPs involves the generation of isolated $\mathrm{Ag} 0$ atoms in the 


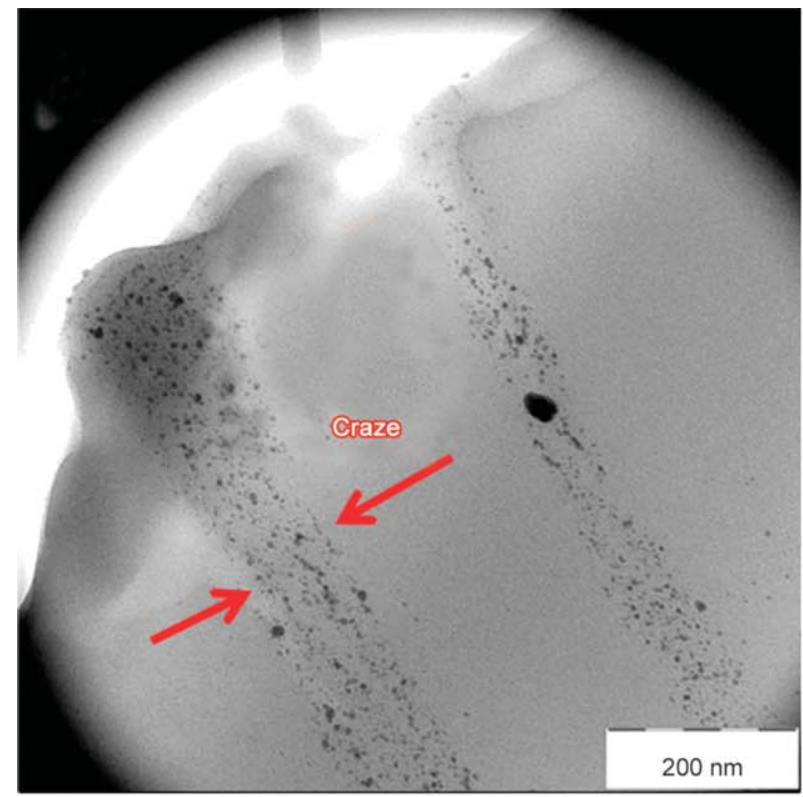

a)

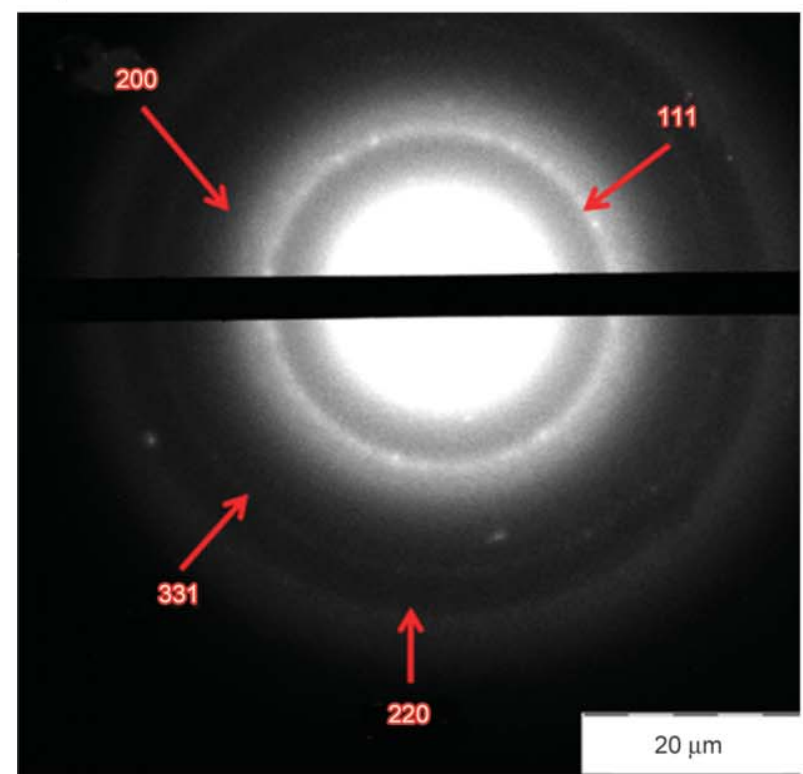

c)

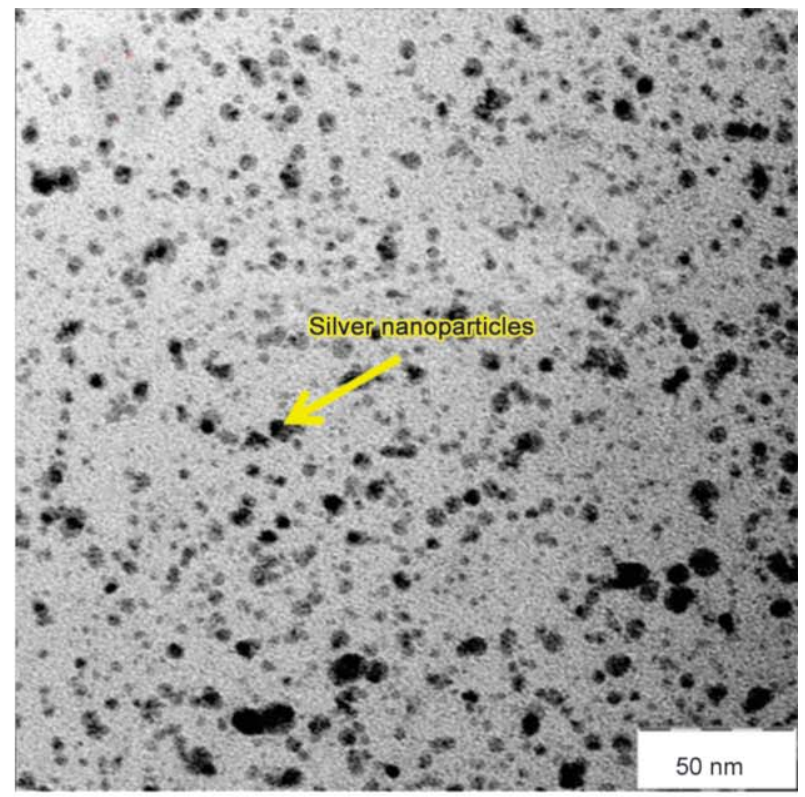

b)

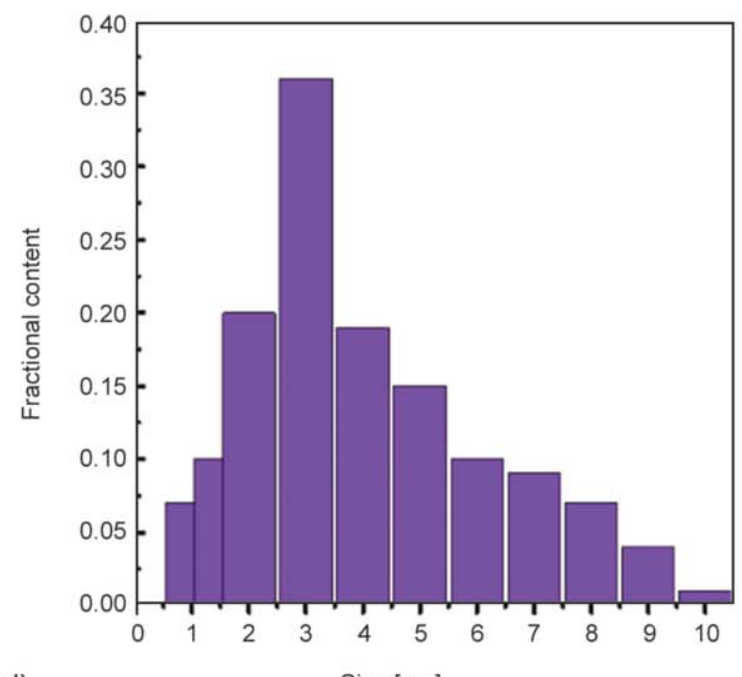

d)

Figure 4. (a, b) TEM images of thin sections of the 15/Ag-PET films containing silver nanoparticles; (c) electron diffractogram of the PET films containing AgNPs; (d) histogram illustrating size distribution of AgNPs. The irradiation time is $15 \mathrm{~min}$.

solution (potential $\mathrm{Ag}+/ \mathrm{Ag}^{0}=-1.8 \mathrm{~V}$ ) [44]. Equation (3) is solely provided by the hydrated electron as a strong reducing agent. Reduction of $\mathrm{Ag}^{+}$on the surface of particles via Equation (5) leads to the enlargement of nanoparticles due to the reduction of the products with lower reduction potentials [40-42], including acetaldehyde [15, 23].

The development of metal NPs in heterogeneous systems is controlled by the contrast of X-ray absorption $[15,22,23]$. As opposed to $\gamma$-radiation or accelerated electrons, the X-ray irradiation dose rate depends on the composition of the environment. Upon irradiation with the 5-BKnV-6W tube, the maximum distribution is provided by the X-ray quanta with an energy of $21 \mathrm{keV}$. Mass absorption coefficients of X-ray irradiation for the elements of the system at $20 \mathrm{keV}$ are the following: for hydrogen, $0.014 \mathrm{~cm}^{2} / \mathrm{g}$; for carbon, $0.224 \mathrm{~cm}^{2} / \mathrm{g}$; for oxygen, $0.617 \mathrm{~cm}^{2} / \mathrm{g}$, and, for silver, $16.95 \mathrm{~cm}^{2} / \mathrm{g}$ [36]. For the irradiated samples, the dose rate can be calculated using the mass absorption coefficients for photons with respect to mass absorption coefficients by Equation (7):

$P=P_{0} \frac{\sum_{\mathrm{i}} W_{\mathrm{i}}\left(\frac{\mu}{\rho}\right)_{\mathrm{i}}}{\left(\frac{\mu}{\rho}\right)_{0}}$ 
where $P$ is the dose rate in the sample; $P_{0}$ is the dose rate in the dosimetric solution; $W_{\mathrm{i}}$ is the mass fraction of the $i^{\text {th }}$ element in the samples $(\mathrm{X}=\mathrm{C}, \mathrm{O}, \mathrm{H}, \mathrm{Ag})$; $(\mu / \rho)_{\mathrm{i}}$ stand for mass absorption coefficients for the elements; $(\mu / \rho)_{0}$ is the mass absorption coefficient of photon energy by the dosimetric solution.

The calculated dose rate $P$ of $\mathrm{X}$-ray irradiation is the following: water-alcohol mixture, $16.23 \mathrm{~Gy} / \mathrm{s}$, PET film, $10.8 \mathrm{~Gy} / \mathrm{s}$, and silver ions in mesopores, $33.6 \mathrm{~Gy} / \mathrm{s}$. Hence, contribution from silver ions to the net absorbed dose rate is maximum. Within mesopores loaded with silver ions, the higher absorbed dose is advantageous for the localized generation of reducing agents; this process is accelerated, and the formation of AgNPs appears to be markedly enhanced. Hence, X-ray irradiation is accompanied by selective radiation-induced chemical processes within mesopores. As irradiation time increases from 10 to $15 \mathrm{~min}$, the absorbed dose increases from 11 to $17 \mathrm{kGy}$, and this value corresponds to the 'safe' sterilization level for polymers when no unwelcome changes in their performance are expected.

Clustering of NPs within mesopores upon X-ray radiolysis depends on the scale of the reactions of reducing agents $[15,22]$. Hydrated electrons can diffuse by a distance of about hundreds of nanometers. Hence, for $100 \mu \mathrm{m}$ thick films, clustering proceeds via local reactions of hydrated electrons generated within mesopores. As the porous structure of crazes is occupied by the aqueous alcohol solution $(>90 \mathrm{wt} \%$ ), acetaldehyde is produced in the surrounding medium. Long-range interphase processes with the participation of acetaldehyde significantly enhance the efficiency of the redox reactions and assist the formation of nanoparticles in the inter-polyelectrolyte complexes [15, 22]. Therefore, long-range diffusion processes provide the conditions for the acetaldehyde-involved reactions, and the growth of NPs in the mesopores of the PET films is intensified.

At the initial stages, atoms and ions exist in specific and unstable states of oxidation. These atoms and intermediate nanoparticles serve as stable nuclei for further nucleation and growth of AgNPs. Formation of stable NPs usually requires several preliminary intermediate stages, which involve the development of unstable small-sized clusters. As the irradiation time increases, this process progresses: metal atoms tend to coalesce into oligomers, which progressively grow into larger clusters. In other words, gradual coalescence of small-sized clusters leads to the build-up of bigger aggregates up to the development of quasimetallic and nanoscale AgNPs [40-42]. Silver atoms $\mathrm{Ag}^{0}$ produced via Equation (3) are involved in the sequence of aggregation events, thus leading to the development of bigger clusters via Equation (4) as shown in Equation (8):

$\mathrm{Ag}^{+}+\mathrm{Ag}^{0} \rightarrow \mathrm{Ag}_{2}{ }^{2+} \ldots \rightarrow \mathrm{Ag}_{8}{ }^{2+} \ldots \rightarrow \mathrm{Ag}_{\mathrm{n}}{ }^{\mathrm{m}}$

Knowing the radius of a silver atom $\left(R_{\mathrm{Ag}}=\right.$ $0.165 \mathrm{~nm})$, the number of silver atoms in each nanoparticle can be calculated as $N=\left(R_{\mathrm{NP}} / R_{\mathrm{at}}\right)^{3}$. When the diameter of the AgNP increases from 2 (10/Ag-PET) to $3 \mathrm{~nm}$ (15/Ag-PET), the number of silver atoms in one nanoparticle increases from $\sim 2 \cdot 10^{2}$ to $\sim 7 \cdot 10^{2}$. The number of active surface silver atoms increases from $\sim 150$ (10/Ag-PET) to $\sim 400$ (15/Ag-PET). Antibacterial efficiency of the silver-containing materials is known to depend on the number of active surface silver atoms, which serve as a source for biocidal silver ions that act as the main force in the fight against bacteria and fungi [34].

\section{Conclusions}

This work presents a facile approach for the synthesis of hybrid organo-inorganic nanomaterials based on PET films via environmental crazing and X-ray radiolysis of silver ions within mesopores under confined conditions. The advantages of this approach for the preparation of HOINs with metallic nanoparticles can be summarized as follows: ecologically safe and reagent-free regime providing an effective reduction of $\mathrm{Ag}^{+}$to AgNPs, formation of AgNPs with dimensions below $5 \mathrm{~nm}$ is accomplished without the use of any capping agents when mesoporous matrix provides confined conditions for in situ reduction of $\mathrm{Ag}^{+}$and serves as a stabilizing medium; all reactions proceed at room temperature with high conversion, this approach is universal for a wide range of polymers (as films, fibers, tapes, yarns, nonwovens, etc.) and metals (including copper, nickel, noble metals, etc. and even bimetallic NPs); the contrast of X-ray absorption provides a selective synthesis of AgNPs (40 kGy) within mesopores, whereas the dose rate for PET matrix (13 kGy) lies within sterilization level; this approach allows pattern reduction of $\mathrm{Ag}^{+}$to AgNPs when X-ray irradiation is performed through a mask. The resultant HOINs with AgNPs are characterized by good mechanical properties and can be used for diverse purposes, including 
antibacterial materials with therapeutic action against gram-positive and gram-negative bacteria and fungi.

\section{Acknowledgements}

This work was financially supported by the Russian Science Foundation (project no. 20-13-00178).

\section{References}

[1] Chauhan B. P.: Hybrid nanomaterials: Synthesis, characterization, and applications. Wiley, Hoboken (2011).

[2] Mobin R., Rangreez T. A., Chisti H. T. N., Inamuddin, Rezakazemi M.: Organic-inorganic hybrid materials and their applications. in 'Functional polymers' (eds.: Mazumder J. M., Sheardown H., Al-Ahmed A.) Springer, Cham, 1135-1156 (2019).

[3] Billah S. M. R.: Textile coatings. in 'Functional polymers' (eds.: Mazumder J. M., Sheardown H., Al-Ahmed A.) Springer, Cham, 825-882 (2019).

[4] Shikha S., Salafi T., Cheng J., Zhang Y.: Versatile design and synthesis of nano-barcodes. Chemical Society Reviews, 46, 7054-7093 (2017). https://doi.org/10.1039/C7CS00271H

[5] Dai J., Bruening M. L.: Catalytic nanoparticles formed by reduction of metal ions in multilayered polyelectrolyte films. Nano Letters, 2, 497-501 (2002).

https://doi.org/10.1021/n10255471

[6] Hundáková M., Pazourková L., Kupková J., Samlíková M., Pazdziora E.: Preparation of antimicrobial polyethylene/inorgano-organo-vermiculite hybrid material. Journal of Nanoscience and Nanotechnology, 16, 7783 7787 (2016). https://doi.org/10.1166/jnn.2016.12552

[7] Jawaid M., Sapuan M. S., Alothman O. Y.: Green biocomposites: Design and applications. Springer, Cham (2017).

[8] Altavilla C., Ciliberto E.: Inorganic nanoparticles: Synthesis, applications, and perspectives. CRC Press, Boca Raton (2017).

[9] Yamaguchi A., Okada I., Fukuoka T., Ishihara M., Sakurai I., Utsumi Y.: Synthesis of nanoparticles through $\mathrm{X}$-ray radiolysis using synchrotron radiation. Nanostructured Thin Films IX, 9929, 992919/1-992919/9 (2016).

https://doi.org/10.1117/12.2237124

[10] Remita H., Remita S.: Metal clusters and nanomaterials: Contribution of radiation chemistry. in 'Recent trends in radiation chemistry' (eds.: Wishart J. F., Rao B. S. M.) World Scientific, Singapore, 347-383 (2010). https://doi.org/10.1142/9789814282093 0013

[11] Belloni J.: Nucleation, growth and properties of nanoclusters studied by radiation chemistry: Application to catalysis. Catalysis Today, 113, 141-156 (2006). https://doi.org/10.1016/j.cattod.2005.11.082
[12] Chmielewski A. G., Chmielewska D. K., Michalik J., Sampa M. H.: Prospects and challenges in application of gamma, electron and ion beams in processing of nanomaterials. Nuclear Instruments and Methods in Physics Research Section B: Beam Interactions with Materials and Atoms, 265, 339-346 (2007).

https://doi.org/10.1016/j.nimb.2007.08.069

[13] Bondaz L., Fontaine P., Muller F., Pantoustier N., Perrin P., Morfin I., Goldmann M., Cousin F.: Controlled synthesis of gold nanoparticles in copolymers nanomolds by X-ray radiolysis. Langmuir, 36, 6132-6144 (2020). https://doi.org/10.1021/acs.langmuir.0c00554

[14] Tung H-T., Chen I-G., Kempson I. M., Song J-M., Liu Y-F., Chen P-W., Hwang W-S., Hwu Y.: Shape-controlled synthesis of silver nanocrystals by X-ray irradiation for inkjet printing. ACS Applied Materials and Interfaces, 4, 5930-5935 (2012).

https://doi.org/10.1021/am3015718

[15] Zezin A. A., Klimov D. I., Zezina E. A., Mkrtchyan K. V., Feldman V. I.: Controlled radiation-chemical synthesis of metal polymer nanocomposites in the films of interpolyelectrolyte complexes: Principles, prospects and implications. Radiation Physics and Chemistry, 169, 108076/1-108076/37 (2020).

https://doi.org/10.1016/j.radphyschem.2018.11.030

[16] Muller F., Fontaine P., Remita S., Fauré M-C., Lacaze E., Goldmann M.: Synthesis of nanostructured metalorganic films: Surface X-ray radiolysis of silver ions using a langmuir monolayer as a template. Langmuir, 20, 4791-4794 (2004).

https://doi.org/10.1021/la049534u

[17] Remita S., Fontaine P., Lacaze E., Borensztein Y., Sellame H., Farha R., Rochas C., Goldmann M.: X-ray radiolysis induced formation of silver nano-particles: A SAXS and UV-visible absorption spectroscopy study. Nuclear Instruments and Methods in Physics Research Section B: Beam Interactions with Materials and Atoms, 263, 436-440 (2007). https://doi.org/10.1016/j.nimb.2007.06.032

[18] Zezin A. A.: Synthesis of hybrid materials in polyelectrolyte matrixes: Control over sizes and spatial organization of metallic nanostructures. Polymer Science Series C, 58, 118-130 (2016). https://doi.org/10.1134/S1811238216010136

[19] Mino L., Bonino V., Picollo F., Fretto M., Agostino A., Truccato M.: Tailoring the local conductivity of $\mathrm{TiO}_{2}$ by X-ray nanobeam irradiation. Advanced Electronic Materials, 5, 1900129/1-1900129/7 (2019). https://doi.org/10.1002/aelm.201900129

[20] Taiji K., Iso Y., Isobe T.: Fluorescent $\mathrm{Ag}^{+}$-exchanged zeolite nanoparticles with improved photoluminescence properties via X-ray irradiation. Journal of Luminescence, 196, 214-220 (2018). https://doi.org/10.1016/j.jlumin.2017.12.028

[21] Clifford D. M., Castano C. E., Rojas J. V.: Highly magnetic Co nanoparticles fabricated by X-ray radiolysis. Radiation Physics and Chemistry, 144, 111-115 (2018). https://doi.org/10.1016/j.radphyschem.2017.11.023 
[22] Feldman V. I., Zezin A. A., Abramchuk S. S., Zezina E. A.: X-ray induced formation of metal nanoparticles from interpolyelectrolyte complexes with copper and silver ions: The radiation-chemical contrast. Journal of Physical Chemistry C, 117, 7286-7293 (2013). https://doi.org/10.1021/jp3090765

[23] Klimov D. I., Zezina E. A., Lipik V. C., Abramchuk S. S., Yaroslavov A. A., Feldman V. I., Sybachin A.V., Spiridonov V. V., Zezin A. A.: Radiation-induced preparation of metal nanostructures in coatings of interpolyelectrolyte complexes. Radiation Physics and Chemistry, 162, 23-30 (2019).

https://doi.org/10.1016/j.radphyschem.2019.04.027

[24] Koetz J., Kosmella S.: Polyelectrolytes and nanoparticles. Springer, Berlin (2007).

[25] Pergushov D. V., Zezin A. A., Zezin A. B., Müller A. H.: Advanced functional structures based on interpolyelectrolyte complexes. Advances in Polymer Science, 255, 173-225 (2014).

https://doi.org/10.1007/12 $2012 \quad 182$

[26] Volynskii A. L., Bakeev N. F.: Solvent crazing of polymers. Elsevier, Amsterdam (1995).

[27] Kinloch A. J., Young R. J.: Fracture behavior of polymers. Springer, Dordrecht (2013).

[28] Arzhakova O. V., Dolgova A. A., Volynskii A. L.: Mesoporous and nanocomposite fibrous materials based on poly(ethylene terephthalate) fibers with high craze density via environmental crazing: Preparation, structure, and applied properties. ACS Applied Materials and Interfaces, 11, 18701-18710 (2019).

https://doi.org/10.1021/acsami.9b02570

[29] Arzhakova O. V., Dolgova A. A., Yarysheva L. M., Volynskii A. L., Bakeev N. F.: Specific features of the environmental crazing of poly(ethylene terephthalate) fibers. Polymer, 56, 256-262 (2015).

https://doi.org/10.1016/j.polymer.2014.11.044

[30] Arzhakova O. V., Dolgova A. A., Yarysheva A. Y., Nikishin I. I., Volynski A. L.: Mechanoresponsive hard elastic materials based on semicrystalline polymers: From preparation to applied properties. ACS Applied Polymer Materials, 2, 2338-2349 (2020).

https://doi.org/10.1021/acsapm.0c00288

[31] Arzhakova O. V., Kopnov A. Y., Nazarov A. I., Dolgova A. A., Volynskii A. L.: 'Green' environmental crazing of polymers in oil-in-water emulsions with high water content. Polymer, 186, 122020/1-122020/11 (2020). https://doi.org/10.1016/j.polymer.2019.122020

[32] Thomas S., Rane A., Abitha V. K., Kanny K., Thomas M. G.: Recycling of polyethylene terephthalate bottles. Elsevier, Oxford (2018).

[33] Sim W., Barnard R. T., Blaskovich M. A. T., Ziora Z. M.: Antimicrobial silver in medicinal and consumer applications: A patent review of the past decade (20072017). Antibiotics, 7, 93/1-93/15 (2018).

https://doi.org/10.3390/antibiotics 7040093
[34] Edwards B.: Silver nanoparticles: Advances in research and applications. Nova, New York (2017).

[35] Tan Y. H., Davis J. A., Fujikawa K., Ganesh N. V., Demchenko A. V., Stine K. J.: Surface area and pore size characteristics of nanoporous gold subjected to thermal, mechanical, or surface modification studied using gas adsorption isotherms, cyclic voltammetry, thermogravimetric analysis, and scanning electron microscopy. Journal of Materials Chemistry, 22, 6733-6745 (2012) https://doi.org/10.1039/c2jm16633j

[36] Hubbell J. H., Seltzer S. M.: Tables of X-ray mass attenuation coefficients and mass energy-absorption coefficients from $1 \mathrm{keV}$ to $20 \mathrm{MeV}$ for elements $Z=1$ to 92 and 48 additional substances of dosimetric interest. Datasheet, NIST Standard Reference Database 126, (1995). https://doi.org/10.18434/T4D01F

[37] Sun Y., Mayers B., Herricks T., Xia Y.: Polyol synthesis of uniform silver nanowires: A plausible growth mechanism and the supporting evidence. Nano Letters, 3, 955-960 (2003). https://doi.org/10.1021/n1034312m

[38] Cheng N. N., Starkewolf Z., Davidson R. A., Sharmah A., Lee C., Lien J., Guo T.: Chemical enhancement by nanomaterials under X-ray irradiation. Journal of the American Chemical Society, 134, 1950-1953 (2012). https://doi.org/10.1021/ja210239k

[39] Kramer E. J.: Microscopic and molecular fundamentals of crazing. in 'Crazing in polymers' (ed.: Kausch H. H.) Heidelberg Springer, Berlin, 1-56 (1983).

https://doi.org/10.1007/BFb0024055

[40] Remita S., Orts J. M., Feliu J. M., Mostafavi M., Delcourt O. M.: STM identification of silver oligomer clusters prepared by radiolysis in aqueous solution. Chemical Physics Letters, 218, 115-121 (1994).

https://doi.org/10.1016/0009-2614(93)E1451-L

[41] Ershov B. G., Janata E., Henglein A.: Growth of silver particles in aqueous solution: Long-lived 'magic' clusters and ionic strength effects. Journal of Physical Chemistry, 97, 339-343 (1993). https://doi.org/10.1021/j100104a013

[42] Mostafavi M., Keghouche N., Delcourt M-O., Belloni J.: Ultra-slow aggregation process for silver clusters of a few atoms in solution. Chemical Physics Letters, 167, 193-197 (1990). https://doi.org/10.1016/0009-2614(90)85004-V

[43] Wardman P.: Reduction potentials of one-electron couples involving free radicals in aqueous solution. Journal of Physical and Chemical Reference Data, 18, 16371755 (1989). https://doi.org/10.1063/1.555843

[44] Henglein A.: The reactivity of silver atoms in aqueous solutions (A $\gamma$-radiolysis study). Berichte der Bunsengesellschaft für physikalische Chemie, 81, 556-561 (1977). https://doi.org/10.1002/bbpc.19770810604 